\title{
Editorial
}

Lynne Cohen

\section{The Medical Devices Adverse Experience Reporting Project}

What do you do when a piece of anaesthetic equipment fails? One essential step, following resolution of the technical emergency, is to report the incident to the proper authorities. One way to ensure they are notified is through the Medical Devices Reporting Project (MDRP), a collaborative effort of the Canadian Medical Association (CMA) and Health and Welfare Canada.

Set up at CMA House in February 1985, the MDRP became fully operational by early summer, receiving between 10 and 12 telephone and mail-in reports a week and forwarding them to the health department's Bureau of Medical Devices (BMD). There, decisions are made as to what, if any, corrective action will be initiated.

As a regulatory agency and the watchdog of medical device manufacturers, the BMD has a huge responsibility in ensuring medical devices sold in Canada are safe when used properly. It does this through a number of means, including pretesting of some instruments. However, since premarket review involves only a tiny proportion of the number of devices available, the BMD must rely heavily on physicians and other health care workers to report adverse experiences. This is why the MDRP was set up.

The Project's mandate includes facilitating the reporting of device problems and promoting the awareness of the need for physicians and other health care workers to be responsible reporters. It involves a nation-wide toll-free telephone hotline (1-800-267-9709), a system for receiving mail-in reports and several programs for promoting medical

Lynne Cohen, Coordinator, Medical Devices Reporting Project, Canadian Medical Association, P.O. Box 8650, Ottawa, Ontario, K1G 0G8. device safety. The project keeps reporters' names in strict confidence, and provides feedback to them on action being taken by the government.

For the purpose of the project, the term "medical device" is not restricted to large electromedical equipment. Indeed, the MDRP has received reports on everything from faulty defibrillators and electronic stethoscopes to weak condoms and similarity of anaesthetic gas cylinder colours. "Medical device" therefore denotes virtually anything used in health care, except drugs.

As anaesthetists are well aware, alerting the federal agency can be crucial in preventing one serious or fatal incident from becoming many. The BMD, through the Health Protection Branch (HPB), can contact users across the country to warn against dangerous devices or device use. Acting promptly when serious problems are brought to its attention, the BMD contacts the manufacturer to direct the kind of resolution required. Further, through the HPB, the BMD issues an "Alert" to hospitals across the country.

Anaesthetists are already familiar with the Medical Device Alerts. They also know that many device hazards become known across Canada only through Alerts. These notices, which have saved many lives, are drawn up only after a problem has been officially filed with the federal government. In other words, by letting the government know about device hazards, health care workers are helping it prevent mishaps from occurring again or somewhere else. By contacting MDRP, this notification can be achieved anonymously and very nearly as quickly as by calling the government directly.

Though potentially lethal device use problems are the most important types of reported incidents, minor problems are also of concern. One being experienced in a particular hospital may also be 
happening in others across the country. When the BMD knows about the problem, the agency can initiate corrective actions to ensure it is permanently resolved.

Though the BMD has been collecting and responding to information about device problems for about six years, officials there have been concerned about the small number of reports being filed about 300 a year. This is why the CMA was approached in 1983.

The sheer size of the medical devices industry suggests this number is inappropriately low. With some 300,000 device classes constituting a $\$ 1.4$ billion-a-year industry in Canada, it is virtually certain many more than 300 incidents are being experienced. One of the problems, it is believed, is that health care workers hesitate to call a regulatory agency. However, it is felt they are not reluctant to call the CMA. The CMA feasibility study conducted in 1983 confirmed what the BMD feared: only a fraction of device problems in Canada is being reported.

It is not the aim of the CMA medical devices project to displace the incident-reporting mechanisms now used in hospitals. These systerns are shaped and upgraded to suit the individual needs of health care centres. What project organizers and supporters would like to see happen is that the MDRP - or the BMD directly - be included as part of the procedures of many more hospitals. Two Ontario hospitals have already written policies which include MDRP in their reporting mechanisms. Such dialogue can only enhance internal systems and result in better health care.

After only ten months of operation, the project is now receiving between eight and ten reports a week. This represents a small increase in the number that was being received by the BMD before the Project was established. Of course, numbers are not the only consideration. A proportion of incidents being reported are about failures of anaesthetic devices, a clear indication of the severity of some of the problems being handled through the MDRP office.

As mentioned, one of MDRP's aims is to heighten awareness in the medical community of the need to use medical devices propertly and safely and of the need to officially report problems that are encountered.
To achieve this, and with the support of the CMA and the BMD, the Project:

- will soon publish a regular newsletter which will include articles on new medical devices on the market;

- arranges lectures about the Project's activities to professional audiences given by CMA members and BMD staff;

- lends support to ongoing educational seminars relating to the hazards of medical devices to be given in universities, hospitals and other professional institutions;

- ensures the toll-free number and objectives of MDRP are repeatedly emphasized in a wide range of medical journals and in other media.

The increased awareness resulting from these activities is no doubt leading to a reduction in mortality and morbidity caused by faulty and misused equipment. And of course, the value of such enhanced public safety and health care is immeasurable.

\section{Le Programme d'information sur les incidents reliés aux instruments médicaux}

Que faire lorsqu'un élément d'équipement d'anesthésie fait défaut? Après avoir résolu le problème technique immédiat, il est essentiel de signaler l'incident aux autorités appropriées. Avoir recours au programme d'information sur les instruments médicaux, qui est le fruit de la collaboration de l'Association médicale canadienne (AMC) et de Santé et Bien-être social Canada, est une façon de $s$ 'assurer que les parties intéressées seront mises au courant.

Établi au siège social de l'AMC en février 1985 , le PIIRIM fonctionnait pleinement au début de 
l'eté. Depuis lors, on y reçoit entre $10 \mathrm{et} 12$ rapports par semaine sous forme d'appels téléphoniques ou de lettres, rapports qui sont transmis au Bureau des instruments médicaux (BIM) du Ministère. C'est à ce stade que l'on décide si des correctifs seront apportés.

À titre d'organisme de réglementation et de surveillance des fabricants d'instruments médicaux, le BIM a l'énorme responsabilité de s'assurer que tous les instruments médicaux vendus au Canada peuvent être employés en toute sécurité. Le Bureau accomplit cette tâche de diverses façons, y compris en procédant à des essais préalables de certains instruments. Cependent, étant donné qu'une faible proportion du nombre d'instruments offerts font l'objet de contrôles précédant leur mise en marché, le BIM doit se fier dans une large mesure à la contribution des médecins et des autre travailleurs de la santé. C'est pour cette raison que le PIIRIM a été établi.

Le Programme a pour objectifs de faciliter la tâche de signaler les problèmes reliés aux instruments médicaux et de faire connaître la nécessité pour les médecins et les autres travailleurs de la santé d'assumer leur rôle de "reporters". Pour ce faire, on a mis en service une ligne téléphonique pan-canadienne sans frais (1-800-267-9709), un mécanisme de traitement des rapports postés et de nombreux programmes d'encouragement de la sécurité dans le domaine des instruments médicaux. Tout le personnel rattaché à ce programme garde rigoureusement secrète l'identité des participants, et leur fournit de la rétroaction sur les mesures prises par le gouvernement.

Aux fins du Programme, l'expression "instruments médicaux" ne désigne pas seulement les gros instruments électromédicaux comme les tomodensitomètres ou les machines d'imagerie par résonance magnétique. De fait, le PIIRIM a reçu des rapports dont l'objet varie des défibrillateurs et des stéthoscopes électroniques défectueux aux préservatifs peu résistants et aux bouteilles de gaz anesthésique dont les couleurs semblaient trop semblables. Autrement dit, "instrument médical" désigne virtuellement tout ce qui est employé dans le domaine des soins de santé, sauf les médicaments.

Comme le savent les anesthésistes, alerter l'organisme fédéral constitue un geste crucial pur empêcher un incident grave ou fatal de se reproduire. Le BIM, par l'entremise de la direction générale de la Protection de la santé (DGPS), peut entrer en contact avec les utilisateurs partout au pays pour les informer de l'aspect dangereux de certains instruments ou de leur emploi. Agissant sans délai lorsque des problèmes graves sont portés à son attention, le BIM prend contact avec le fabricant concerné pour mettre au point la solution qui s'impose. En outre, toujours par l'entremise de la DGPS, le BIM diffuse une "alerte" dans tous le hôpitaux du pays.

Les anesthésistes connaissent déjà ces "alertes". Ils savent également que de nombreux dangers reliés à des instruments ne sont connus à l'échelle du pays que grâce à ces alertes. Ces avis, qui ont permis de sauver de nombreuses vies, ne sont émis qu'après qu'un problème ait été officiellement porté à l'attention du gouvernement fédéral. En d'autres mots, en mettant le gouvernement au courant des dangers reliés à l'emploi d'un instrument médical, les travailleurs de la santé l'aident à empêcher qu'un incident ne se reproduise ailleurs. Grâce au PIIRIM, cela peut être fait de façon anonyme et presque aussi rapidement que si l'on prenait directement contact avec l'organisme gouvernemental.

Bien que l'aspect potentiellement mortel des problèmes d'emploi d'un instrument constitue le plus important type d'incident signale, les problèmes mineurs n'en sont pas moins dénués d'intérêt. Un incident survenu dans un hôpital en particulier peut aussi se produire ailleurs au pays. Une fois que le BIM a pris connaissance du problème, il peut prendre les correctifs qui s'imposent pour apporter une solution définitive.

Bien que le BIM recueille des renseignements concernant les problèmes reliés aux instruments médicaux et prenne les mesures qui s'imposent depuis presque six ans, ses responsables s'inquiètent du nombre réduit de rapports, soit 300 par année. C'est pour cette raison que des démarches ont été entreprises auprès de l'AMC en 1983.

La seule ampleur de l'industrie des instruments médicaux permet de déduire que ce nombre est trop faible. Pour cette industrie de quelque 300000 catégories d'instruments representant un chiffre d'affaires annuel de 1,4 milliard de dollars au Canada, il est presque certain que le nombre d'incidents reliés à ces produits est supérieur à 300 . 
On croit que l'un des facteurs de ce nombre réduit serait que les travailleurs de la santé hésitent à prendre contact avec un organisme de réglementation. Cependant, on pense qu'ils seraient moins réticents à communiquer avec l'AMC. En fait, une étude de faisabilité tenue en 1983 pour le compte de l'AMC a confirmé les craintes du BIM : seule une fraction des problèmes reliés aux instruments médicaux au Canada fait l'objet d'un rapport.

Le Programme d'information sur les incidents reliés aux instruments médicaux de l'AMC n'a pas pour objet de se substituer aux mécanismes d'information actuellement en cuvre dans les hôpitaux. Ces mécanismes sont conçus et mis à jour en fonction des besoins particuliers des centres de soins de santé. Cependant, les organisateurs du programme et ceux qui l'appuient aimeraient bien que le PIIRIM - ou le BIM, directement - soit intégré au processus d'information sur les incidents reliés aux instruments médicaux dans un nombre accru d'hôpitaux. Deux hôpitaux ontariens ont déjà élaboré des lignes de conduite dans lesquelles le PIIRIM est intégré à leurs mécanismes d'information. Une telle mesure ne peut qu'améliorer les pratiques internes et produire un degré supérieur de soins de santé.

Après seulement dix mois d'existence du Programme, on y reçoit entre huit et dix rapports par semaine. Cela représente une légère augmentation par rapport au nombre de rapports que recevait le BIM avant la mise en cuvre du Programme. Bien entendu, il n' $q$ a pas que les chiffres qui comptent. En effet, une proportion des incidents signalés sont reliés à des instruments anesthésiques, ce qui constitue une indication inéfutable de la gravité de certains des problèmes traités dans les bureaux du PIIRIM.

Comme on l'a mentionné précédemment, un des objectifs du PIIRIM consiste à sensibiliser davantage les membres de la communauté médicale quant au besoin d'employer les instruments médicaux de façon appropriée et sûre, et de signaler officiellement les problèmes qui surviennent.

À cette fin, sous les auspices de Programme et avec l'appui de l'AMC et du BIM,

- on publiera bientôt un bulletin périodique qui renfermera des articles sur les nouveaux instruments médicaux mis sur le marché.

- on élaborera des exposés sur les activités entreprises dans le cadre du Programme. Ces exposés seront donnés par des membres de l'AMC et du personnel du BIM à des auditoires composés de professionnels.

- on contribuera au déroulement de séminaires de formation permanente sur les dangers associés aux instruments médicaux. Ces séminaires se tiendront dans des universités, des hôpitaux et d'autres institutions à caractère professionnel.

- on s'assurera que le numéro de la ligne téléphonique sans frais et les objectifs du PIIRIM seront mis en évidence de façon soutenue dans une vaste gamme de publications médicales et dans d'autre médias.

Il est indubitable que le degré accru de sensibilité résultant de ces activités mènera à un réduction de la mortalité et de la morbidité imputables au matériel défectueux et mal employé. On ne saurait trop insister sur la valeur inestimable d'une telle amélioration de la sécurité du public et de la qualité des soins médicaux. 